\title{
Sufficient conditions for the discrimination of motion*
}

\author{
HERBERT H. BELL† and JOSEPH S. LAPPIN \\ Vanderbilt University, Nashville, Tennessee 3724C
}

\begin{abstract}
When one half of a randomly contoured pattern is displaced in one of four directions between successive exposures, Ss are capable of discriminating the direction of the displacement. Accuracy of discrimination decreases as a function of the relative displacement within the pattern, independent of the visual angle subtended by the pattern. Supplementary data suggest that Ss are unrealistically confident in the accuracy of their discrimination. The results demonstrate that identification of specific contours is not necessary for the discrimination of motion and suggest that some type of correlational process is employed by the visual system in dealing with spatially and temporally displaced patterns.
\end{abstract}

Apparent (or stroboscopic) motion may be perceived when two or more simple stimuli are presented in rapid temporal succession at slightly different spatial locations. Within certain ranges of exposure duration, stimulus intensity, and temporal succession, the stimulus sequence appears to consist of a single stimulus pattern moving continuously within the visual field (see Graham, 1965).

The traditional approach to the study of apparent motion has utilized the S's report of the subjective quality of the perceived motion for simple stimulus patterns presented within a homogeneous visual field. There are two major difficulties with this method of investigation. First, apparent motion is a tenuous phenomenon. The exact combination of spatial and temporal values required to produce the subjective report of optimal apparent motion varies widely among Ss, presumably reflecting the contribution of the decision criteria employed by the Ss in describing the phenomenon (Kahneman, 1967). Second, when a simple form is presented within a homogeneous visual field at two discernibly different spatial and temporal positions, the perception of motion may be based on a logical inference from the spatial and temporal characteristics of the display and a willingness to report motion-the perception of motion per se is not required (Kinchla \& Allan, 1969).

One technique for solving these methodological problems is to ask the $S$ to discriminate the direction of motion within a complex random dot pattern. By the use of these stimuli, the salient cues provided by two easily recognizable forms at different spatial and temporal locations can be removed from the stimulus display. This technique is analogous to that originated

*This work was supported by Public Health Service Grant MH 21105-01. We are grateful to Kenneth Disch and John Eriksen for their assistance in generating the stimuli, to the Department of Nuclear Medicine for the use of their computer facilities, and to Janet Briscoe and Sharon Likely for their assistance in conducting the experiment.

† Requests for reprints may be sent to Herbert H. Bell, Department of Psychology, Vanderbilt University, Nashville, Tennessee 37240 . by Julesz for the study of stereopsis and has been applied by Julesz and others to the problem of motion perception. Much of the previous research is summarized by Julesz (1971). The present experiment differs from this previous research in two important respects. First, the behavioral indicator was discrimination of the direction of displacement rather than a subjective report about the appearance or quality of the motion. Second, unlike a number of studies summarized by Julesz, only two sequential stimulus patterns were presented on each trial.

Discrimination of the direction of displacement probably reflects different underlying processes than does the subjective report of apparent motion. A failure to report the direction of displacement correctly does not necessarily result in a failure to report the subjective experience of motion. Anstis (1970) has reported subjective impressions of motion in the direction opposite the actual physical displacement as a result of reversing the brightness values of each element within a pair of random dot patterns. Indeed, Julesz (1971, p. 205) emphasizes that if two successive random dot patterns were generated independently of each other and then presented within an apparent motion paradigm, the subjective experience of motion could occur merely on the basis of attending to a few local elements in the two patterns. Anstis (1970) and Julesz (1971) have emphasized that the phenomenal report of apparent motion may be obtained even when the correlation, or structural similarity, between the successive stimuli is minimal. Obviously, whenever a pattern is spatially displaced within the appropriate spatial and temporal constraints, the phenomenal report of apparent motion may be obtained. Our operations are not designed to assess the phenomenal aspects of apparent motion, but rather the ability of the human $S$ to report accurately the direction of a spatial displacement between two successively presented random dot patterns.

The main objective of this experiment was to determine if the displacement of a visual pattern was sufficient for the discrimination of the direction of displacement in the absence of a clearly defined form. The perception of motion has been frequently linked 
Fiest stimulus

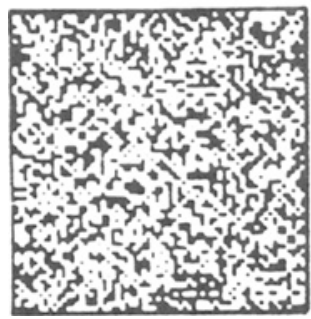

SECOND STIMULUS

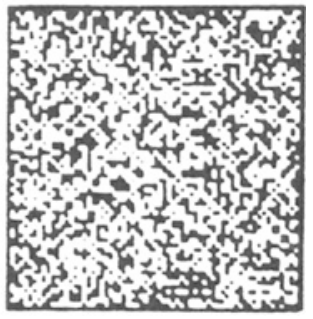

Fig. 1. Photograph of a pair of stimulus patterns. The left half of the second stimulus has been displaced two columns to the left. In the experiment, these patterns were presented sequentially and were superimposed.

with the perception of form (Titchener, 1902; Kolers, 1972). Indeed, the reports of Julesz (1971) and Anstis (1970) suggest that Ss tend to report a spatial change within a visual pattern as motion, even when the change does not involve a coherent displacement of pattern elements. Hence, in order to get a more precise specification of the visual system's ability to utilize spatial and temporal information from sequentially presented stimuli, it is necessary to employ a forced-choice indicator-e.g., the direction of the displacement.

The methodological distinction between the presentation of only two sequential patterns and the repetitive presentation of apparent motion stimuli is also important in assessing the visual system's ability to discriminate motion. A number of Julesz's studies have employed movies (cinematograms) in which the random dot patterns are repeated numerous times. There may well be a difference in performance as a function of the number of times a motion sequence is repeated. Doherty and Keeley (1972) demonstrate that in general the accuracy of form identification is directly related to the number of successive presentations of the same brief visual stimulus. It is logically possible for a similar type of effect to occur when the stimuli are presented via an extended cinematogram in which the consecutive frames portray a successive or repeating sequence of displacements. Under these circumstances, the direction of the displacement may be perceived at any point within the film sequence-not necessarily after the presentation of the initial displacement.

A more important distinction between single and multiple presentations of random dot patterns is based on the nature of the cues provided. Suppose one were to generate a cinematogram in which a camouflaged square was successively displaced within a random dot display. The first two presentations (frames) within the cinematogram might do nothing more than identify two areas of difference or form within the display (Julesz \& Hesse, 1970; Lappin \& Bell, 1972). Beginning with the third presentation, the perception of motion could occur merely on the basis of a perceivable form occupying successive spatial positions with the passage of time. As
Julesz (1971) emphasizes, the mere use of random dot patterns as stimuli does not by itself guarantee the removal of all form cues for the perception of motion.

The detection of a displacement within a random dot pattern has been demonstrated by Pollack (1972). But the detection of a change in a pattern does not imply that the direction of displacement has been perceived: It is sufficient for the $\mathbf{S}$ to detect a difference in the two successive patterns-either that an element in the first pattern has been deleted from the second or that a new element has been added to the second pattern-but not necessary that he match the corresponding segments of the two displaced patterns. As Pollack made clear, conclusions about the perception of motion are not strictly required by such a demonstration.

To summarize, we know of no unequivocal evidence that the visual system is capable of matching two displaced complex random patterns and thereby recognizing the direction of the displacement. Indeed, the requisite correlational process is inconsistent with the common conception of vision as an integrative system that acts to fuse stimuli presented in the same spatial position at adjacent points in time (see Kahneman. 1968).

The main objective of this experiment was to determine whether the displacement of a visual pattern was sufficient for discriminating the direction of motion. The question is whether the presence of a distinct form is necessary for the discrimination of the direction of motion. The basis of this investigation is the use of random dot patterns to preclude matching the two successive patterns by distinct local contours. The use of complex random dot patterns requires that the visual system correlate two globally defined patterns.

A second purpose of the study was to determine the dependence of this discrimination upon the extent of displacement of the second pattern. Two alternative ways of measuring the amount of displacement are in relation to the grain of the pattern and in relation to distance on the retina. The appropriateness of these two measures was compared by displacing part of a random matrix by one of three numbers of rows or columns at either of two visual angles.

\section{MAIN EXPERIMENT}

\section{Method}

\section{Stimuli}

The stimulus patterns were 64 by 64 matrices of black and white squares photographed from an oscilloscope display. These patterns were generated randomly with two constraints; each column contained 32 black squares and the right and left halves of each row contained 16 black squares. The stimuli were produced by generating the first pattern and then shifting either the left or right half of this pattern in one of four directions (up, down, left, or right) by either 2,4 , or 8 squares. A photograph of the first stimulus and second stimulus in which the left half has been shifted two squares to the left is shown in Fig. 1. 
The stimuli were presented via $16-\mathrm{mm}$ film under the control of a modified stop-action movje projector (Traid 614) and additional timing equipment. A stimulus presentation consisted of a pair of stimulus patterns sequentially presented and a preand postexposure field containing a centered fixation point. Each stimulus pattern was represented by a single frame and was exposed for $300 \mathrm{msec}$. These stimuli were rear-projected at eye level on a screen located approximately $80 \mathrm{~cm}$ from the S. Two projector locations were selected so that the visual angle subtended by the patterns was either 4 or $8 \mathrm{deg}$ square. The average luminance of the stimuli at both visual angles was approximately $15.5 \mathrm{~cd} / \mathrm{m}^{2}$. The interstimulus interval was approximately $5 \mathrm{msec}$ and was determined by the length of time required for the projector to advance the film to the next frame with the projector speed set at 24 frames/sec.

\section{Procedure}

Fach of the six Ss served in two sessions. A session contained eight blocks of 24 trials, with each block consisting of a different sequence of the 24 possible combinations of the four directions of displacement. three extents of displacement, and two displaced halves. One block of trials was obtained by photographing the basic set of 24 stimulus pairs, and three additional blocks were obtained by photographing the basic set of 24 stimulus pairs at 90,180 , and $270 \mathrm{deg}$ of rotation. At the 0 - and 180-deg orientations. the right or left half of the pattern was displaced on each trial, while at the 90 - and 270 -deg orientations, either the top or bottom half was displaced. This sequence of four film blocks was run in one direction for the first four blocks of trials and in the opposite direction for the last four blocks of trials. The ordering of the visual angles and the direction in which the film was run were counterbalanced across Ss.

The Ss viewed the film binocularly and were required to identify the direction of displacement (right, left, up, or down). Prior to viewing the film at each visual angle, each $S$ was given 12 practice trials on which he was informed of the actual direction of displacement after each response. During the data-collection phase of the experiment. no feedback was provided, but the $\mathrm{S}$ was given the total number of correct discriminations after each block of 24 trials.

\section{Subjects}

The six Ss were the first author and five volunteer graduate students at Vanderbilt with no previous training in similar experiments. All Ss had normal or corrected-to-normal vision.

\section{Results and Discussion}

The results are summarized in Table 1, which gives the percentage of correct discriminations made by each $\mathrm{S}$ for each combination of relative displacement and visual angle. The data have been combined across sessions, film blocks, and directions of film presentation because
Table 1

Percentage of Correct Identifications of Direction of Motion as a Function of the Relative Displacement (Number of Rows or Columns of the Matrix) and the Retinal Size of the Pattern (Height and Width in Degrees of Visual Angle)

\begin{tabular}{|c|c|c|c|c|c|c|c|c|}
\hline \multirow{2}{*}{$\begin{array}{l}\text { Visual } \\
\text { Angle }\end{array}$} & \multirow{2}{*}{$\begin{array}{c}\text { Relative } \\
\text { Displace- } \\
\text { ment }\end{array}$} & \multicolumn{7}{|c|}{ Subject } \\
\hline & & BB & $\mathrm{HB}$ & DB & $\mathrm{KD}$ & $\mathrm{SE}$ & WH & Average \\
\hline 4 Deg & $\begin{array}{l}2 \\
4 \\
8\end{array}$ & $\begin{array}{l}94 \\
55 \\
23\end{array}$ & $\begin{array}{l}97 \\
59 \\
22\end{array}$ & $\begin{array}{l}94 \\
53 \\
39\end{array}$ & $\begin{array}{l}94 \\
75 \\
28\end{array}$ & $\begin{array}{l}91 \\
52 \\
41\end{array}$ & $\begin{array}{l}89 \\
34 \\
33\end{array}$ & $\begin{array}{l}93 \\
55 \\
31\end{array}$ \\
\hline 8 Deg & $\begin{array}{l}2 \\
4 \\
8\end{array}$ & $\begin{array}{l}89 \\
42 \\
30\end{array}$ & $\begin{array}{l}88 \\
34 \\
39\end{array}$ & $\begin{array}{l}91 \\
42 \\
33\end{array}$ & $\begin{array}{l}98 \\
56 \\
25\end{array}$ & $\begin{array}{l}97 \\
53 \\
41\end{array}$ & $\begin{array}{l}89 \\
42 \\
36\end{array}$ & $\begin{array}{l}92 \\
45 \\
34\end{array}$ \\
\hline
\end{tabular}

separate analyses showed these variables to have no consistent effect on the discrimination accuracy and no interaction with the other variables. As may be seen, discrimination accuracy decreased as the relative extent of the displacement increased, independent of the retinal distance of the displacement.

In order to further describe the discrimination among the four directions of motion, 4 by 4 confusion matrices were constructed, one for each of the relative extents of displacement. Table 2 gives a measure of the discriminative distance $[-1 \mathrm{n} \eta$ from Luce's choice theory $^{1}$ (see Luce, 1963)] between each pair of directions of motion. As may be seen, discrimination was poorer between the two vertical directions (up vs down) than between the two horizontal directions (right vs left) or between the four pairs of horizontal vs vertical directions. For displacements of either four or eight elements, the vertical discriminations were no better than chance, although the other discriminations continued above chance at displacements of eight elements.

Analysis of the confusion matrices also indicated a consistent bias to report the direction of displacement as "up." One measure of this bias ${ }^{1}$ is given in Table 3. This bias was evident in every condition and for every S; "up" was the most frequent response in 80 of the total $96 \mathrm{~S}$ by Condition combinations. An explanation for this bias is not at all clear, especially in view of the poor discrimination characteristic of vertical displacements.

To summarize, small displacements are sufficient for human Ss to discriminate the direction of motion of a complex random pattern that is devoid of the positional cues provided by the contours of a distinct form. The

Table 2

Discrimination Distances ( $-1 \mathrm{n} \eta)$ Among the Four Directions of Displacement at Each Extent of Displacement Relative Extent of Displacement

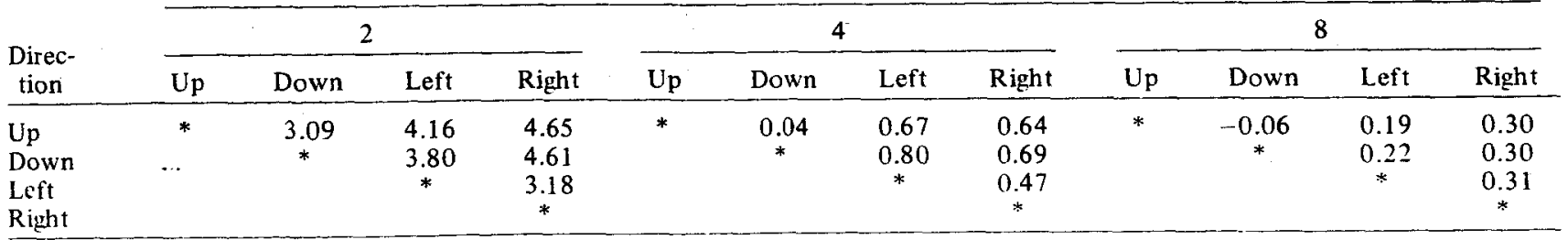


Table 3

Response Biases 1 for Each Relative Extent of Displacement

\begin{tabular}{llll} 
& \multicolumn{3}{c}{ Relative Extent of Displacement } \\
\cline { 2 - 4 } Response & 2 & 4 & 8 \\
\hline Up & .33 & .33 & .35 \\
Down & .17 & .18 & .18 \\
Left & .28 & .25 & .23 \\
Right & .26 & .24 & .23 \\
\hline
\end{tabular}

accuracy of these discriminations was found to decrease sharply with the relative extent of these displacements, as measured by the rows and columns of the random matrix, but was independent of the two angular distances of these displacements on the retina. Discrimination accuracy also depended to a lesser degree upon the specific direction of displacement, being poorer for displacements in a vertical direction. Additionally, the occurrence of biases for reporting movement in an upward direction suggests a discrepancy between the subjective impressions of motion and the capability for discrimination of motion in the conditions of this experiment.

\section{SUPPLEMENTARY EXPERIMENT}

The purpose of this experiment was to investigate the apparent discrepancy between the subjective experience of motion and the discrimination between directions of displacement: The subjective experience of motion is often compelling, even when the judgments of direction are very inaccurate. Ss in the main experiment were consistently confident in their directional judgments and were typically surprised at the number of errors they made with the larger displacements. Additionally, there were strong tendencies to experience the motion in certain directions more than in others. The illusory nature of these perceptions has persisted for the authors, even after considerable experience in producing and viewing the stimuli and in scoring other S's judgments. In fact, our initial pilot work in selecting experimental conditions was quite misled by unfounded confidence in our ability to discriminate the direction of displacement.

The specific objective of this supplementary experiment was to examine the correspondence between Ss' confidence ratings, the accuracy of their judgments, and the extent of the displacement. We had planned to continue this experiment for several sessions, eventually providing feedback after each response, but equipment failure curtailed the experiment after one session for each of three Ss.

\section{Method}

\section{Subjects}

Three undergraduates from Vanderbilt University served as paid volunteers. All Ss had normal or corrected-to-normal vision.

\section{Stimuli and Procedure}

The film sequence was the same as in the main experiment. The stimuli were presented under the same procedures as in the main experiment, with the following exceptions: (1) The size of the patterns was constant at $4 \mathrm{deg}$ of visual angle; (2) feedback was provided only during the 12 practice trials at the beginning of the session; and (3) Ss were instructed to rate their confidence in the correctness of their discrimination along a 5-point scale, with 0 representing no confidence and 4 representing comple te certainty in the direction of the displacement.

\section{Results and Discussion}

Table 4 gives the percentage of correct responses and the average confidence rating associated with each extent of displacement. As in the main experiment, the discrimination accuracy falls off sharply with increases in the extent of displacement. The confidence ratings, however, remained quite high for all three extents of displacement. The discrimination accuracy associated with the two highest confidence ratings is also given in Table 4; the fact that the accuracy on these selected trials is heavily dependent upon the extent of displacement and is approximately the same as on all trials with all confidence ratings indicates that these confidence ratings were only weakly correlated with the accuracy of the Ss' judgments of direction.

A more specific analysis of the correlation between discrimination accuracy, confidence ratings, and extent of displacement was obtained by computing the amount of information transmitted between accuracy (correct vs incorrect) and confidence rating and between accuracy and extent of displacement. This statistic provides an appropriate measure of the correlation between these pairs of variables because it assumes only a nominal scale for each variable (see Attneave, 1959). Table 5 gives these two values of information transmitted for each of the three Ss; in all three cases, the correlation between accuracy and extent of displacement is slightly higher than between accuracy and confidence. In other words, the $E$, simply by knowing the extent of displacement, and accuracy as a function of displacement, was better able to predict whether the S's judgment was correct or incorrect than was the $S$ who had just observed the stimulus and made his response!

Table 4

Percentage of Correct Responses, Average Confidence Rating, and Percentage of Correct Responses at the Two Highest Levels of Confidence for Each Extent of Displacement

\begin{tabular}{cccc}
$\begin{array}{c}\text { Extent } \\
\text { of } \\
\begin{array}{c}\text { Displace- } \\
\text { ment }\end{array}\end{array}$ & $\begin{array}{c}\text { Percen- } \\
\text { tage } \\
\text { Correct }\end{array}$ & $\begin{array}{c}\text { Average } \\
\text { Confi- } \\
\text { dence } \\
\text { Rating }\end{array}$ & $\begin{array}{c}\text { Percentage } \\
\text { Correct } \\
\text { at Two } \\
\text { Highest } \\
\text { Confidence } \\
\text { Levels }\end{array}$ \\
\hline 2 & 75 & 3.6 & 78 \\
4 & 48 & 3.0 & 44 \\
8 & 30 & 2.7 & 31 \\
\hline
\end{tabular}


Although small response biases were obtained in this experiment, they were inconsistent with the biases in the first experiment.

The results of this experiment demonstrate the validity of the premise in the introduction to this report that the subjective experience of motion does not imply an ability to discriminate the direction of displacement.

Questions about what variables determine the quality of the subjective experience of motion and about whether confidence ratings can be trained to reflect reliably the discrimination accuracy remain for further research.

\section{DISCUSSION}

This study demonstrates that a small displacement provides sufficient information for discriminating the direction of motion. The salient cues provided by the simple contours of a distinct form are not necessary for this discrimination, nor are more than two brief presentations of a pattern necessary. Moreover, the perception of motion has here been assessed by an objective measure of discrimination accuracy rather than by the Ss' subjective reports of an experience of motion.

In order to discriminate the direction of displacement correctly, the visual system must be capable of matching the corresponding segments of two successive patterns presented at different points in space and must also be capable of registering the difference in spatial positions of the two patterns. Such discriminations would be impossible if the visual system were merely to combine successive stimuli by a simple additive process. The present demonstration that something is required beyond an additive process extends other recent evidence of a differentiation of sequential patterns (Lappin \& Bell, 1972) and evidence of the stereoscopic cross-correlation of spatially disparate patterns dichoptically presented to each eye (see Julesz, 1971). The present demonstration would seem to require that spatially and temporally displaced visual patterns can be brought into correspondence by a correlational process. One wonders whether this correlational process might be the same one that produces stereopsis. Interestingly, both phenomena exhibit a greater sensitivity to horizontal than to vertical displacements.

The discrimination of the direction of displacement depended upon the extent of the displacement relative to the grain of the pattern; surprisingly, performance was independent of the absolute extent of displacement on the retina, at least for the two angular sizes used in this experiment. Certainly, however, there must be boundary conditions under which discrimination depends upon retinal displacement. At present, we are unable to make a good guess as to what these limiting conditions might be.

Concerning the temporal parameters that are critical for this phenomenon, we can hazard some guesses on the basis of initial pilot work. Since Lappin \& Bell (1972)
Table 5

Amount of Information Transmitted Between the Extent of Displacement and Accuracy $[H(D ; A)]$ and Between Accuracy and Confidence $[H(C ; A)]$ for Each $S$

\begin{tabular}{lcc}
\hline & \multicolumn{1}{c}{ Information Transmitted (Bits) } \\
\cline { 2 - 3 } S & H(D;A) & H(C;A) \\
\hline AC & 0.07 & 0.04 \\
AS & 0.12 & 0.05 \\
DL & 0.12 & 0.05 \\
\hline
\end{tabular}

found that the detection of a difference between two successive patterns fell near a chance level by an ISI of only $30 \mathrm{msec}$, it seems very unlikely that the correlational process involved in the present experiments could operate over a longer interval. We can also guess from brief pilot work that exposures of less than about $200 \mathrm{msec}$ produce a decrease in accuracy and that accuracy does not change as the exposure is lengthened beyond this duration.

One final point concerns contrasts between the methods for studying the perception of motion. The present experiments clearly indicate that discrimination accuracy and subjective reports of motion reflect different underlying processes. Which behavioral indicator should be used depends, of course, upon the question being asked, but there has been surprisingly little use of discrimination accuracy as a behavioral indicator in studies of motion perception. We would suggest that there is need for much wider use of discrimination measures.

\section{REFERENCES}

Anstis, S. M. Phi movement as a subtractive process. Vision Research, 1970, 10, 1411-1430.

Attneave, F. Applications of information theory to psychology. New York: Holt, Rinehart \& Winston, 1959.

Doherty, M. E., \& Keeley, S. M. On the identification of repeatedly presented, brief visual stimuli. Psychological Bulletin, 1972, 78, 142-154.

Graham, C. H. Perception of movement. In C. H. Graham (Ed.), Vision and visual perception. New York: Wiley, 1965.

Julesz, B. Foundations of cyclopean perception. Chicago: University of Chicago. 1971.

Julesz, B., \& Hesse, R. I. Inability to perceive the direction of rotational movement of line segments. Nature, 1970. 225. 243-244.

Kahneman, D. An onset-onset law for one case of apparent motion and metacontrast. Perception \& Psychophysics. 1967. 2, 557-583.

Kahneman, D. Method, findings, and theory in studies of visual masking. Psychological Bulletin, 1968, 70, 404-425.

Kinchla, R. A., \& Allan, L. G. A theory of visual movement perception. Psychological Review, 1969. 76, 537-558.

Kolers, P. A. Aspects of motion perception. Oxford: Pergamon Press, 1972.

Lappin. J. S., \& Bell. H. H. Perceptual differentiation of sequencial visual patterns. Perception \& Psychophysics. 1972. 12. 129-134.

luce, R. D. Detection and recognition. In R. D. Luce, R. R. 
Bush, and E. Galanter (Eds.). Handbook of mathematical psychology. Vol. I. New York: Wiley, 1963.

Pollack, I. Detection of changes in spatial position: Short-term visual memory or motion perception? Perception \& Psychophysics, 1972, 11, 17-27.

Titchener, E. B. An outline of psychology. New York: Macmillan, 1902.

\section{NOTE}

1. Luce's (see 1963) model of choice behavior in a discrimination experiment may be represented by the following equation

$$
p_{i j}=\frac{\eta_{i j} \beta_{i}}{\sum_{i^{\prime}}\left(\eta_{i^{\prime} j} \beta_{i^{\prime}}\right)}
$$

where $p_{i j}$ is the probability of response $i$ given that stimulus $j$ was presented, $\beta_{i}$ is a response bias such that

$$
\sum_{\mathbf{i}} \beta_{\mathbf{i}}=1
$$

and $\eta_{\mathrm{ij}}$ is a measure of the perceptual similarity of stimuli $i$ and $j$ such that $\eta_{\mathrm{ij}}=\eta_{\mathrm{ji}}, \eta_{\mathrm{ij}}=1$, and $0 \leqslant \eta_{\mathrm{ij}} \leqslant 1$. This latter parameter is estimated from the data by the following equation

$$
\eta_{i j}=\left[\frac{p_{i j}}{p_{j j}} \cdot \frac{p_{j i}}{p_{i i j}}\right]^{1 / 2}
$$

and the logarithmic transformation $-\log _{\mathrm{e}} \eta_{\mathrm{ij}}$ may be shown to provide a measure of the discriminative "distance" between the stimuli $i$ and $j$. For readers not familiar with this measure of discrimination, its interpretation is very similar to the measure $d^{\prime}$ in signal detection theory (see Luce, 1963).

(Received for publication December 22, 1972; revision received March 6, 1973.) 